\title{
Reliability Assessment of Satellite Ni-Cd Battery based on the End of Discharge Voltage
}

\author{
Cui-ying Duan,Guang Jin,Jing-lun Zhou,Tian-yu Liu, Lang Zhang \\ College of Information System and Management, National University of Defense Technology, \\ Changsha, Hunan, P.R. China, 410073. \\ E-mail: dcyyspa@sohu.com
}

Keywords: Ni-Cd battery, reliability assessment, performance degradation, pseudo life, random effect regression

\begin{abstract}
Ni-Cd battery is the key component of the satellite energy system with the characteristics of long life and high reliability, and few or no failure occurs in its traditional life testing. In this paper, based on the performance degradation modeling method, we establish a dynamic degradation model to capture the evolution of the end of discharge voltage (EODV), which is a function of cycle number and temperature. Two methods are utilized to assess the reliability of Ni-Cd battery. Results show that the random effect regression method obtains a more conservative reliability estimation result that the pseudo life based method does.
\end{abstract}

\section{Introduction}

The Ni-Cd battery is one of the important components of the satellite and its reliability directly influences the reliability of the satellite. As for a product of long-life and high reliability, there often will be few failures or even "zero-failure" in the conventional life test and accelerated life test of the Ni-Cd battery [1]. The traditional reliability assessment methods of the batteries are usually based on the failure lifetime [2][3]. However, for the reason of long-life and high reliability, it is very difficult to obtain the failure time of the Ni-Cd battery by using life test or accelerated life test. In such case, the performance degradation modeling method makes it possible to evaluate the reliability of the Ni-Cd battery [4][5].

In this paper, we investigate the reliability assessment of the Ni-Cd battery. By studying the cycle life test data of a certain type of Ni-Cd battery, we find that the end of discharge voltage (EODV) is a key indicator which can reflect the performance of a Ni-Cd battery. During the cycle life test, EODV declines gradually with cycles, and shows a strong correlation with the ambient temperature. Firstly, we establish a dynamic degradation model of the EODV as the function of cycle number and temperature using the performance degradation modeling method. Based on the dynamic degradation model, we successfully predict the cycle life of the Ni-Cd battery. Then, the reliability of the Ni-Cd battery is evaluated based on the pseudo life and degradation path distribution, respectively.

\section{Cycle life test for Ni-Cd battery}

Nine Ni-Cd batteries connected in series are used to conduct the cycle life test to study their performance degradation over charge/discharge cycles. The total rated capacity of the nine Ni-Cd batteries is $80 \mathrm{Ah}$ and the nominal voltage of a single battery is $1.2 \mathrm{~V}$. The depth of discharge (DOD) is set to be $15 \%$ to simulate the actual working process on the satellite. During the test, the EODV of each battery and the average temperature in each cycle are recorded by the computer. Testing results are shown in Figure 1. 


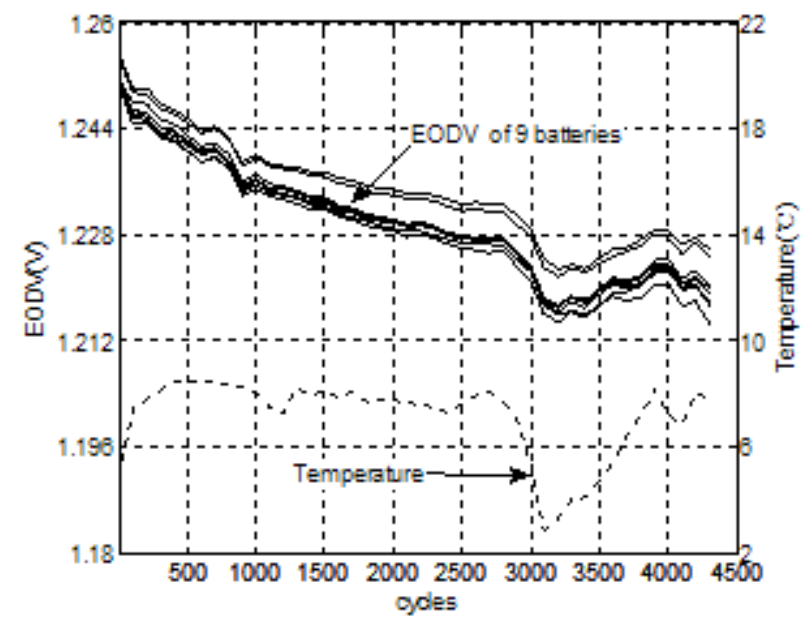

Figure. 1 EODV and Temperature variation vs cycles

It is clear that the EODV of each battery degrades as cycle increases. EODV fade will affect the Ni-Cd working performance seriously. When the EODV decreases below a critical value, a battery cannot supply enough energy and fails. However, except for the cycles, temperature also affects the EODV apparently. The EODV of a battery in a cycle will be quite low when the cycle temperature is low, but it can go back to a high level in the next cycle if the temperature increases. Thus the EODV degradation model must consider the temperature effect.

\section{Ni-Cd EODV degradation model considering temperature effect}

J. Paatero [6] proposed a model of the Ni-Cd battery, which can describe the quantitative relation between EODV and temperature. In this model, temperature affects EODV by a linear term plus an exponential term,

$$
\operatorname{EODV}(T)=a+c \cdot T+d \cdot \exp (T)
$$

Where $\mathrm{T}$ is the temperature and a, c, $\mathrm{d}$ are the parameters of the model need to be evaluated.

Note that the model in (1) does not consider the degradation of the battery. In practical applications, the EODV of the Ni-Cd battery degrades as the cycle number increases. Because the variation range of the temperature in our test is quite small, we assume that the temperature stress will not affect the degradation rates of the battery. Through the cycle life test of the Ni-Cd battery, we find that the EODV of the battery almost degrades linearly as the cycle number increases without considering the temperature influence. Thus, the EODV degradation model under time-varying temperature stress can be formulated as

$$
\operatorname{EODV}(t, T)=a+b \cdot t+c \cdot T+d \cdot \exp (T)
$$

where $t$ is the cycle number, $T$ is the temperature and $a, b, c, d$ are the parameters of the model need to be evaluated.

\section{Reliability Assessment of Ni-Cd Battery}

\section{Reliability Assessment based on Pseudo Life}

The pseudo life based reliability assessment method is quite popular in engineering [7]. The step of this method is as follows:

(1) Establish a degradation model to acquire the characteristics of the key degradation indictor.

(2) Estimate model parameters with the degradation data of each sample.

(3) Extrapolate the pseudo life of each sample with the degradation parameter estimators and a pre-defined threshold. 
(4) Test which distribution the pseudo life fits best.

(5) Calculate the reliability indices and draw the reliability curves with the life distribution.

According to the demand of the Ni-Cd battery for satellite, a single battery is considered as ineffective when its EODV is smaller than a threshold Df, which is called failure threshold. Under the temperature $\mathrm{T}$, the cycle life of the battery $\mathrm{L}$ can be predicted using the following function:

$$
L=\left[D_{f}-a-c \cdot T-d \cdot \exp (T)\right] / b
$$

Note that the cycle life above is called pseudo life because this life is the extrapolation life, not the real life of the battery.

The parameter estimation of the degradation models in Eq. (2) can be obtained easily with the regression technique. According to the engineering experience, the threshold is set to be $0.8 \mathrm{~V}$ at the temperature $6^{\circ} \mathrm{C}$. The pseudo life of the 9 batteries can be calculated based on Eq. (3), and the results are shown in Table 1.

Table 1. Pseudo life of the $9 \mathrm{Ni}-\mathrm{Cd}$ batteries

\begin{tabular}{c|c|c|c|c|c}
\hline \hline Battery index & 1 & 2 & 3 & 4 & 5 \\
\hline Pseudo life(10 cycles) & 10.23 & 12.11 & 11.85 & 9.40 & 12.97 \\
\hline Battery index & 6 & 7 & 8 & 9 & \\
\hline Pseudo life(10 $10^{4}$ cycles) & 10.51 & 9.00 & 8.40 & 12.00 & \\
\hline \hline
\end{tabular}

The life distribution of the electronic product is usually Weibull distribution [8]. Through the hypothesis test, it is found that the pseudo life of a single Ni-Cd battery approximately obeys the Weibull distribution. Therefore, we use the Weibull distribution in the regression of the single battery life. The probability density function and the reliability function of the Weibull distribution are

$$
\left\{\begin{array}{l}
f(t)=\frac{m}{\eta}\left(\frac{t}{\eta}\right)^{m-1} \exp \left\{-\left(\frac{t}{\eta}\right)^{m}\right\} \\
R(t)=\exp \left\{-\left(\frac{t}{\eta}\right)^{m}\right\}
\end{array}\right.
$$

With the pseudo life in Table 2, the maximum likelihood estimation (MLE) of the Weibull distribution is

$$
\hat{\eta}=113592, \hat{m}=8.35 \text {. }
$$

Finally, the reliability indices such as the mean time to failure (MTTF) and q-percentile life Tq are easy to calculate based on the life distribution. Bootstrap resampling method is utilized to calculate the interval estimation of the reliability indices aforementioned [9]. The point and interval estimation of the reliability indices are shown in Table 2 and the reliability curve is shown in Figure 2.

Table 2. Point and interval estimation of the MTBF and $\mathrm{T}_{\mathrm{q}}$ based on the pseudo life method

\begin{tabular}{c|c|c}
\hline \hline Reliability indices & Point $\left(10^{4}\right)$ & $90 \%$ intervals $\left(10^{4}\right)$ \\
\hline$M T B F$ & 10.6 & {$[9.6,11.6]$} \\
\hline$T_{0.5}$ & 10.7 & {$[9.7,11.7]$} \\
\hline$T_{0.8}$ & 9.4 & {$[8.3,10.8]$} \\
\hline$T_{0.9}$ & 8.6 & {$[7.5,10.3]$} \\
\hline \hline
\end{tabular}




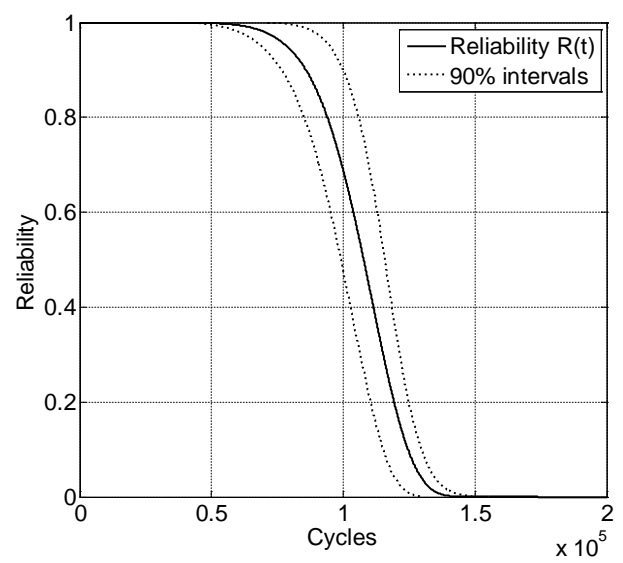

Figure 2 Reliability curve of the Ni-Cd battery based on pseudo life method

\section{Reliability Assessment based on random effect regression method}

The random effect regression method assumes the parameters of the degradation model is a random vector following the multi-dimensional normal distribution, which can describe the item-toitem variations as result of difference in materials and manufacturing processes [10].

Assume that the EODV measurement at time tij on unit $i$ is yij $(i=1, \ldots, 9$ and $j=1, \ldots, m)$, $m$ is the total cycles in the cycle life test, then the random effect regression model is

$y_{i j}=D_{i j}+v_{i j}$

Where the actual degradation path is

$D_{i j}=a_{i}+b_{i} t_{i j}+c_{i} T_{i j}+d_{i} \exp \left(T_{i j}\right)$

where Tij is the temperature at time tij, $v_{i j}$ is the random error which follows a normal distribution with shape parameter 0 and scale parameter $\sigma$, and the parameter vector follows a multidimensional normal distribution with mean $\mu$ and covariance $\Sigma$.

Collecting into $Y_{i}=\left(y_{i 1}, y_{i 2}, \cdots, y_{i m}\right)^{\mathrm{T}}$ the observations from unit $\mathrm{i}$, we can obtain the equivalent expression of the random effect regression model as follows:

$Y_{i}=X_{i}(\mu+\theta)+\varepsilon_{i}$

Where

$$
\begin{gathered}
X_{i}=\left(\begin{array}{cccc}
1 & t_{i 1} & T_{i 1} & \exp \left(T_{i 1}\right) \\
\vdots & \vdots & \vdots & \vdots \\
1 & t_{i m} & T_{i m} & \exp \left(T_{i m}\right)
\end{array}\right), \\
\theta \sim \operatorname{MVN}(0, \Sigma), \varepsilon_{i} \sim \operatorname{MVN}\left(0, \sigma^{2} I_{m}\right) .
\end{gathered}
$$

Thus, the observation vector $Y_{i}$ follows a multi-dimensional normal distribution with mean $E\left(Y_{i}\right)$ and covariance $\operatorname{Var}\left(Y_{i}\right)$, wherein

$$
\left\{\begin{array}{l}
E\left(Y_{i}\right)=X_{i} \mu \\
\operatorname{Var}\left(Y_{i}\right)=X_{i} \Sigma X_{i}^{\mathrm{T}}+\sigma^{2} I_{m}
\end{array}\right.
$$

The MLE of unknown parameter ${ }^{\mu}, \Sigma$ and $\sigma$ can be obtained by maximizing the total log likelihood function of the $9 \mathrm{Ni}-\mathrm{Cd}$ batteries which is expressed as

$L=\sum_{i=1}^{n}\left\{-\frac{1}{2} \log \left[\operatorname{det}\left(\operatorname{Var}\left(Y_{i}\right)\right)\right]-\frac{1}{2}\left[Y_{i}-E\left(Y_{i}\right)\right]^{\mathrm{T}}\left[\operatorname{Var}\left(Y_{i}\right)\right]^{-1}\left[Y_{i}-E\left(Y_{i}\right)\right]\right\}$

With the parameter estimation, we can conduct the reliability estimation of the Ni-Cd battery by a simulation method with the following steps: 
(1) Resample a parameter vector of the degradation model in Eq. (6) from the multi-dimensional normal distribution $M V N(\mu, \Sigma)$ and extrapolate the corresponding pseudo life with a predefined temperature $6^{\circ} \mathrm{C}$ and threshold $0.8 \mathrm{~V}$.

(2) Repeat step (1) N (N=50000) times and obtain $\mathrm{N}$ pseudo lives $L_{1}, L_{2}, \cdots, L_{N}$.

(3) The reliability function can be expressed as

$$
R(t)=P(L \geq t)=\frac{\text { number of }\left\{L_{i} \geq t\right\}}{N} .
$$

(4) Calculate the reliability indices MTTF and Tq based on the reliability function R(t).

According the random effect regression method, the point and interval estimation of the reliability indices are shown in Table 3 and the reliability curve is shown in Figure 3.

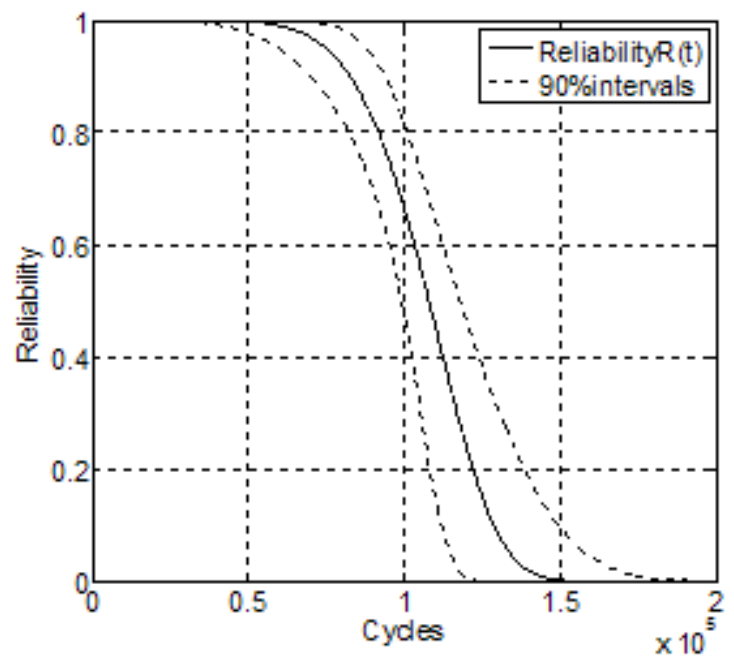

Figure 3 Reliability curve of the Ni-Cd battery based on random effect regression method Table3 Point and interval estimation of the MTBF and Tq based on random effect regression method

\begin{tabular}{c|c|c}
\hline \hline Reliability indices & Point $\left(10^{4}\right)$ & $90 \%$ intervals $\left(10^{4}\right)$ \\
\hline$M T B F$ & 10.6 & {$[9.7,11.7]$} \\
\hline$T_{0.5}$ & 10.7 & {$[9.8,11.8]$} \\
\hline$T_{0.8}$ & 9.2 & {$[8.1,10.1]$} \\
\hline$T_{0.9}$ & 8.2 & {$[6.9,9.4]$} \\
\hline \hline
\end{tabular}

\section{Conclusion}

In this paper, we establish a dynamic degradation model of the EODV of the Ni-Cd battery using the performance degradation modeling method. After that, we evaluate the reliability of the Ni-Cd battery based on the pseudo life method and the random effect regression method respectively. The results of the reliability indices point estimation and the interval estimation obtained by the two methods are basically consistent. More specifically, the reliability assessment result based on the random effect regression method is a little more conservative than that based on the pseudo life method. The method used in this paper can be extended to the life prediction and reliability assessment of other long lifetime products.

\section{REFERENCES}

1. J Eliason. Ni-Cd battery reliability for shuttle serviced spacecraft. The 10th IECEC, 1975:12971306.

2. ST Tseng, ZC Wen. Step-Stress Accelerated Degradation Analysis for Highly Reliability Products. Journal of Quality Technology, 2000, 32(3): 209-216. 
3. W Nelson. Accelerated Life Testing Step-Stress Model and Data Analyses. IEEE Transactions on Reliability, 1980, 29(2): 103-108.

4. Jayaram. Reliability prediction through degradation data modeling using a quasi-likelihood approach. PARMS, 2005:193-199.

5. V Crk. Reliability assessment from degradation data. Proceedings Annual Reliability and Maintainability Symposium, 2000: 155-161

6. J Paatero. A mathematical model for flooded nickel cadmium battery, Helsinki University of Technology, Department of Physics, Tech. Rep, 1997.

7. AM Deng, X Chen. Reliability Assessment Based on Performance Degradation Data, Journal of Astronautic, Vol.27, No.3, 546-552, 2006.

8. DNP Murthy, Xie M, RY Jiang. Weibull Models, New York, Wiley, 2003.

9. B Efron. Bootstrap Confidence Intervals for a Class of Parametric Problems. Biometrika, 1985, 72: 45-58.

10. XX Yuan, M Pandey. A nonlinear mixed-effects model for degradation data obtained from inservice inspections. Reliability Engineering and System Safety, 2009, 94: 509-519. 